Laser Chem. 1988, Vol. 9, pp. 27-46

(C) 1988 Harwood Academic Publishers GmbH

Photocopying permitted by license only

Reprints available directly from the Publisher

Printed in the United Kingdom

\title{
Two-dimensional Imaging of Photofragments $\dagger$
}

\author{
JOHN W. THOMAN, JR., and DAVID W. CHANDLER \\ Combustion Research Facility, Sandia National Laboratories, Livermore, \\ California 94550 \\ DAVID H. PARKER \\ Chemistry Department, University of California, Santa Cruz, California \\ 95064
}

MAURICE H. M. JANSSEN

Physics Department, Catholic University, Nijmegen, Toernooiveld, 6525 ED, Nijmegen, The Netherlands

(Received January 15, 1988; in final form March 9, 1988)

The technique of photofragment imaging is described, and several examples of the power of the technique are presented. Two-dimensional images of state-selected photofragments from the photodissociations of $\mathrm{CD}_{3} \mathrm{I}$ and $\mathrm{H}_{2} \mathrm{~S}$ illustrate how photofragment imaging reveals $\beta$ parameters, brancing ratios, Doppler profiles and vector correlations. Comparisons are made with Doppler profiling and one-dimensional time-of-flight techniques.

KEY WORDS: Photodissociation; photofragment imaging.

\section{INTRODUCTION}

Photodissociation is one of the simplest laser-induced chemical processes. Laser photodissociation has been studied for many years because it is a way to initiate a unimolecular reaction with some control

$\dagger$ Research sponsored by the US Department of Energy, Office of Basic Energy Sciences, Division of Chemical Sciences. 
over the starting conditions. Using an ultraviolet laser to access a dissociative electronic state allows the experimenter to place a known quantity of energy into a system and to follow a reaction by monitoring the products.

To understand the dissociation dynamics of a reaction, one would like to know as much as possible about the photofragments produced. The most important question is what is the molecular structure of the fragments, but for small molecules the answer is often obvious. On these smaller systems it is feasible to investigate the internal states of the photofragments, their velocities and the correlations between these parameters. Techniques have been developed to measure the angular distribution, ${ }^{1-3}$ the recoil velocity, ${ }^{4-6}$ and the internal energy of fragments produced. ${ }^{7-9}$ Doppler profiling techniques have been utilized to study the correlation between the fragments' velocities and internal energies. ${ }^{10-12}$ Multiphoton ionization (MPI) time-of-flight (TOF) techniqus have been demonstrated that give the equivalent information obtained by the Doppler techniques without the use of sub-Dopplerlinewidth lasers. ${ }^{13}$ These techniques involve the one-dimensional projection of the three-dimensional distribution of particles. One demonstration of a two-dimensional optical imaging technique has been reported whereby the photofragments are detected with laser-induced fluorescence. ${ }^{14}$ This implementation was limited to low spatial resolution by the small number of detector elements employed. In this paper, we describe a two-dimensional imaging technique wherein photofragments are tagged by resonance-enhanced multiphoton ionization (REMPI) and imaged onto a two-dimensional, position-sensitive detector. ${ }^{15} \mathrm{We}$ refer to this technique as photofragment imaging.

Conceptually, photofragment imaging involves photolysis of a low pressure gas of a parent molecule with a linearly polarized laser to create a point source of photofragments. Before the fragments have time to recoil from the laser focus region, a second laser selectively ionizes a single quantum state of one of the fragments. Since the mass of an electron is much smaller than that of the fragment, the ions retain the vector velocity distribution of the parent neutral fragments. The ion distribution expands to a conveniently measurable size, and is projected onto a two-dimensional detector using an electric field. Wiley and McLaren have discussed the experimental conditions appropriate for a TOF apparatus. ${ }^{16}$

The position at which the ions strike the two-dimensional detector is 
sensitive to the velocity with which the neutral fragments recoil in the photodissociation process. We place the detector parallel to the symmetry axis of the dissociation and use a numerical inversion technique ${ }^{17}$ to reconstruct the three dimensional velocity distribution of the photofragments. Photofragment imaging has a multiplexing advantage in that the entire distribution of photofragments (in a single quantum state which is selected by the ionization laser) is sampled in one laser shot. The detection system has a single ion sensitivity, and signal averaging over a few thousand laser shots produces clear images.

One limitation common to photofragment imaging and to onedimensional TOF imaging is Coulomb distortion. The ionization laser ejects electrons and leaves a small group of positively charged ions. If there are too many ions produced per laser shot (much greater than 1000 for our experimental conditions), they will mutually repel and add an additional velocity component to each ion. A discussion of this effect is presented in conjunction with the thoughtful REMPI study by Black and Powis of the photodissociation of methyl iodide. ${ }^{18}$ In photofragment imaging, this distortion is readily apparent as an instability in the diameter of the image. Since this Coulomb distortion is easily detected, it may be corrected by reducing the laser fluence in real time.

What information do we gain from photofragment images? Photofragment imaging provides all of the information obtainable from Doppler or one-dimensional TOF techniques. In several instances, photofragment imaging is a more sensitive technique, or less susceptible to error. Photofragment imaging is a more sensitive technique, or less susceptible to error. Photofragment images are sensitive to: the relative polarization of the photolysis laser $(\varepsilon)$ with respect to the transition dipole moment $(\mu)$ of the parent molecule; the vector velocities ( $\mathbf{v})$ of the photofragments; the range of photofragment velocity components probed by the ionization laser; the relative polarizations of the two laser beams with respect to the detector; and the vector correlations of $\varepsilon, \mathbf{v}, \mu$ and $\mathbf{J}$ (the rotation of the fragment). These will each be demonstrated and discussed in this paper.

\section{EXPERIMENTAL}

The technique of photofragment imaging has been described previously. ${ }^{15} \mathrm{~A}$ brief outline is provided here, along with some extensions 
of the technique. A schematic of the experimental apparatus appears in Figure 1. The parent molecule of interest $\left(\mathrm{CD}_{3} \mathrm{I}\right.$ or $\left.\mathrm{H}_{2} \mathrm{~S}\right)$ is seeded in 1 to 6 bar of helium and delivered through a pulsed molecular beam to a high vacuum chamber. A linearly polarized photolysis-laser pulse is loosely focused in the molecular beam. The ideal source of photofragments is a point source; in these experiments, the source is defined by the intersection of the molecular beam and the photolysis laser. The source is a cylinder of $\sim 2 \mathrm{~mm}$ length and $\sim 0.5 \mathrm{~mm}$ diameter. The finite length contributes to a broadening of the images along one axis. A second linearly polarized laser is fired collinear and counterpropagating to the first laser after a small time delay (10-20 ns). Both lasers travel perpendicular to the molecular beam. The second (or "probe") laser state selectively ionizes fragments from the dissociation by REMPI.

A repeller field of $\sim 100 \mathrm{~V} / \mathrm{cm}$ accelerates the ions along the molecular beam axis. The ions travel along a TOF tube and are detected by a two-dimensional position sensitive detector positioned normal to the flight-tube axis. Since a skimmed molecular beam is used, the molecules carry very little $(\sim 10 \mathrm{~K})$ velocity perpendicular to the detector axis. We choose the detector axis coincident with the molecular beam axis so that the initial velocity of the parent molecules does not effect the images observed. The detector consists of a microchannel plate in front of a fast (50-ns lifetime) phosphor screen. A pulsed-voltage shutter screen placed immediately in front of the detector rejects ions with $\mathrm{m} / \mathrm{e}$ greater than the ion being imaged. Although this shutter grid greatly reduces the background signal at the center of the detector, it limits the resolution of the apparatus by introducing a mesh over the images. The detection system is being redesigned to minimize this problem.

The images that appear on the phosphor screen are recorded with a digital charge-coupled device (CCD) camera (Photometrics 200 thermoelectrically cooled electronic camera with a Thompson-CSF CCD that has a resolution of $384 \times 576$ pixels) and stored on a microcomputer. A digital recording system allows for signal averaging over long periods of time, background subtraction, signal processing, and a variety of analysis and data reduction techniques. A pixel by pixel summation along either the rows or the columns of the image produces the equivalent of the Doppler profile of the ions. One image may be summed in two different ways (once over rows and once over columns) 


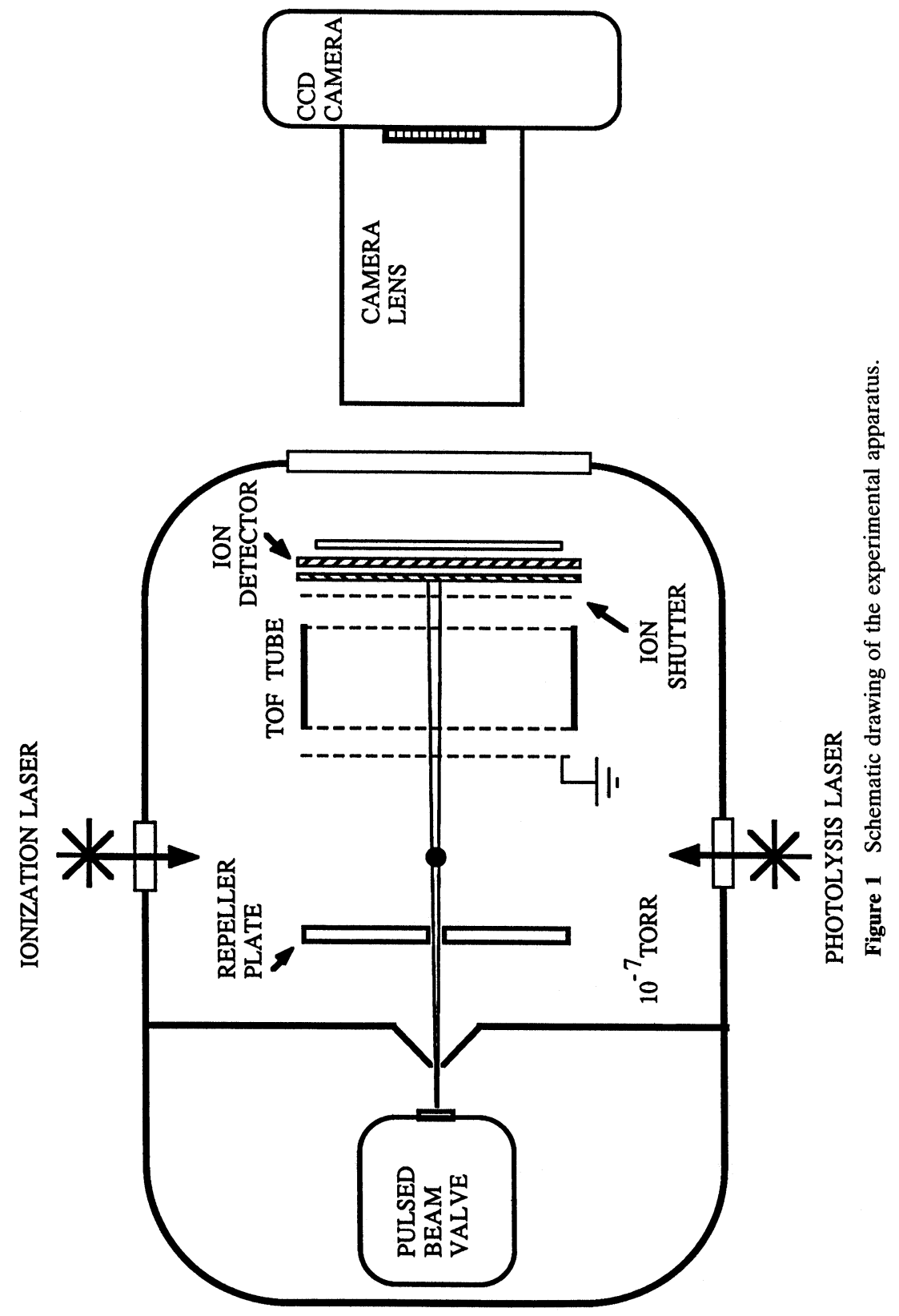


to give two Doppler profiles corresponding to two orthogonal components of the velocity distribution. Thus photofragment imaging has a multiplexing advantage of two over one-dimensional TOF techniques.

For the studies of $\mathrm{H}_{2} \mathrm{~S}$ photodissociation, a few modifications were made to the photofragment imaging technique. A single laser is used both to photodissociate $\mathrm{H}_{2} \mathrm{~S}$ and to ionize $\mathrm{H}$ atoms by $2+1$ REMPI through the H Lyman- $\alpha$ transition. This naturally forces the photolysis and probe laser polarizations to be the same.

The Doppler profile of the $\mathrm{H}$ atom is larger than the ionization laser's bandwidth. Several research groups have taken advantage of the relatively large Doppler width of $\mathrm{H}$ atom fragments to derive information concerning the dynamics of the dissociation. ${ }^{19,20}$ For Doppler profiling techniques, a large Doppler width is an advantage because it permits one to collect more data points to define the Doppler profile for a given bandwidth laser.

For photofragment imaging (and for one-dimensional TOF techniques) a large Doppler width is a disadvantage. The ionization laser must cover the entire Doppler profile. Ideally one would like a laser with a top-hat profile in the frequency domain that completely encompasses the Doppler profile of the species of interest. The "ideal laser" is approximated in the imaging experiments by scanning the frequency of the ionization laser while the image is being collected. Because the entire image is collected for each laser shot, scanning the laser is equivalent to adding up images produced with a fixed frequency laser at several different wavelengths. A scanned laser will produce nearly the equivalent of a top-hat frequency profile for the probe laser. In the case where one scanned laser functions as both the photolysis and probe lasers, one must implicitly assume that the dissociation dynamics do not change over the range of frequencies scanned.

\section{RESULTS AND DISCUSSION}

\section{Limiting cases of photofragment distributions}

Photofragment imaging is sensitive to a variety of experimental and dynamical parameters as described in the introduction. In order to develop a feeling for the images produced we examine two limiting 
photodissociation cases. First we consider the photodissociation of $\mathrm{CD}_{3} \mathrm{I}$ at $266 \mathrm{~nm}$ and the imaging of $\mathrm{CD}_{3}$ radicals via REMPI through the $3 p^{2} A_{2}^{\prime \prime}-2 p^{2} A_{2}^{\prime \prime}$ transition. ${ }^{21}$ Some data from this experiment is reported elsewhere. ${ }^{22} \mathrm{Next}$, we examine the dissociation of $\mathrm{H}_{2} \mathrm{~S}$ using a single laser pulse at the energy equivalent to $1 / 2$ the Lyman- $\alpha$ transition in atomic hydrogen. This pulse dissociates $\mathrm{H}_{2} \mathrm{~S}$ in one step and also ionizes $\mathrm{H}$ atoms via $2+1$ REMPI. These two photodissociation cases were chosen because they occur on very fast time scales; ${ }^{6,23,24}$ thus rotation of the parent molecular during the dissociation will not wash out $\varepsilon-\mu-\mathbf{v}$ correlation seen in the images.

The distribution of photofragments has been considered in detail by many authors. ${ }^{25-29}$ Typically, photofragment distributions are characterized by the $\beta$ parameter in the equation:

$$
I(\theta)=\left[1+\beta P_{2}(\cos \theta)\right] / 4 \pi,
$$

where $\theta$ is the angle relative to the polarization vector of the photolysis laser and $P_{2}$ is the second order Legendre polynomial. Loo et al. have measured a $\beta$ parameter of $1.8 \pm 0.1$ for $266-\mathrm{nm}$ dissociation of $\mathrm{CD}_{3} \mathrm{I}$ to produce $\mathrm{CD}_{3}+\mathrm{I} .{ }^{13} \mathrm{~A} \beta$ parameter of 2.0 is expected for a pure parallel transition with instantaneous dissociation. In contrast, the 248-nm photodissociation of $\mathrm{H}_{2} \mathrm{~S}$ to produce $\mathrm{H}+\mathrm{SH}_{\text {has a measured }}{ }^{24} \beta$ parameter of $-0.66 \pm 0.06$, where $\beta=-1.0$ is expected for a pure perpendicular transition with instantaneous dissociation. Photodissociation of $\mathrm{H}_{2} \mathrm{~S}$ at $243 \mathrm{~nm}$ should be comparable to $248-\mathrm{nm}$ photodissociation, based on the results of van Veen et al. ${ }^{24}$

The fragments produced in a single quantum state from a photodissociation will lie on nested spheres. Each sphere is correlated with a single quantum state of the sibling fragment. The radius of a given sphere depends on the translational energy of the fragments and the time between photolysis and viewing. Figure 2 illustrates the distribution of photofragments seen for these two limiting cases. The right hand side of Figure 2 shows an artist's schematic of the threedimensional distributions, and the left hand side shows experimental two-dimensional images.

The limiting case of a parallel transition is shown in Figures $2 \mathrm{a}$ and $2 \mathrm{~b}$ for $\mathrm{CD}_{3} \mathrm{I}$ photolysis. The number density of fragments, which is represented by the darkness in the three-dimensional schematic drawings in Figure 2, is proportional to $\cos ^{2} \theta$, where $\theta$ is the angle to the polarization vector, $\varepsilon$. In three dimensions, the photofragment dis- 


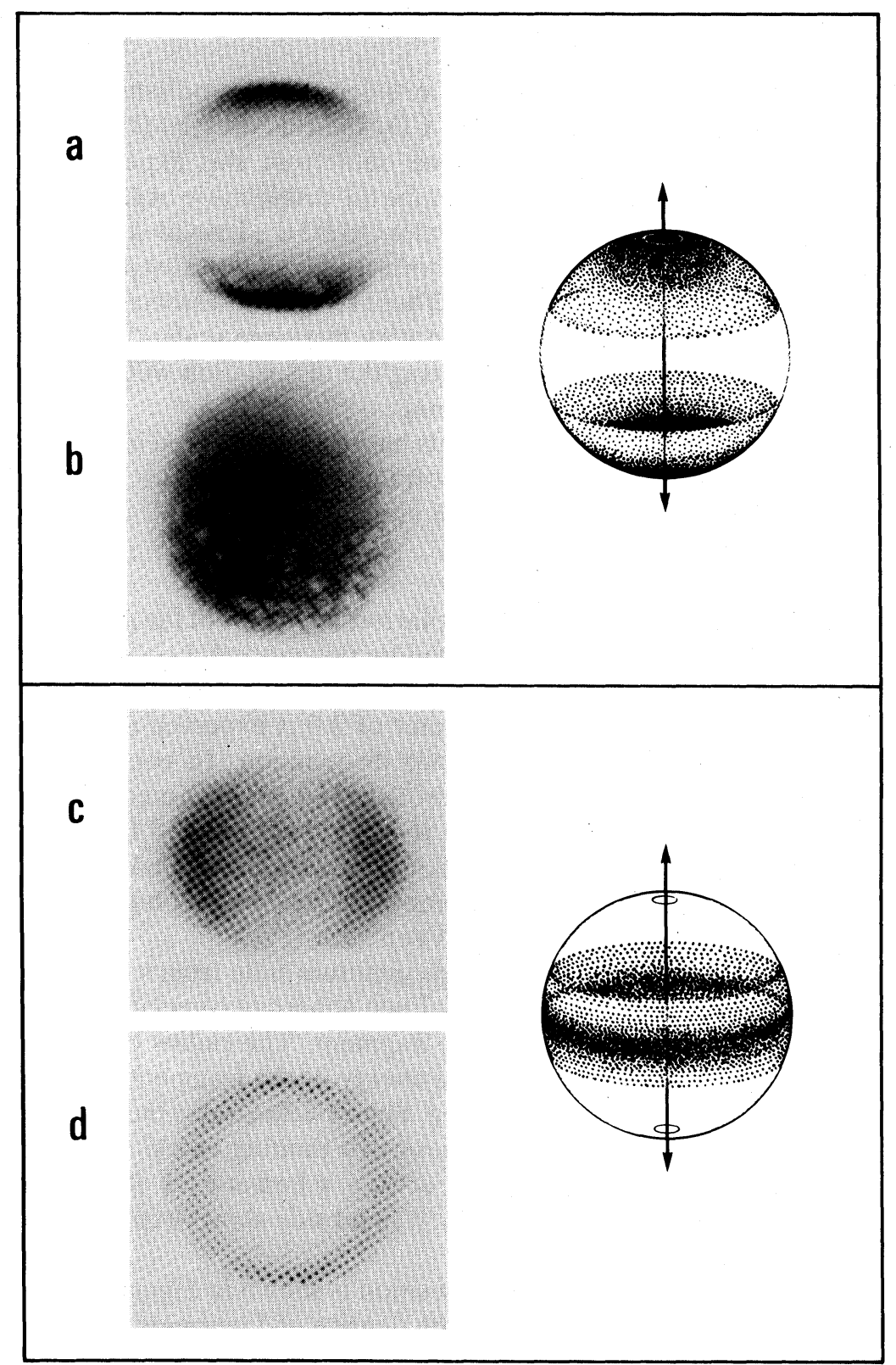

Figure 2 Experimental two-dimensional images and artist's schematic threedimensional representations of the limiting cases of photofragment distributions. A double headed arrow represents the polarization vector of the photolysis laser. (a) A parallel transition (the transition dipole, $\mu$, is parallel to the symmetry axis of the molecule) viewed with the polarization vector of the photolysis laser, $\varepsilon$, perpendicular to the detector axis. Image of $\mathrm{CD}_{3}$ radicals from 266-nm photolysis of $\mathrm{CD}_{3} \mathrm{I} . \mathrm{CD}_{3}$ radicals are ionized by $2+1$ REMPI through the $\mathrm{Q}$ branch of the $0_{0}^{0}$ transition. (b) A parallel transition with $\varepsilon$ parallel to the detector axis, using the same system as in (a). (c) A perpendicular transition with polarization as in (a). Image of $\mathrm{H}$ atoms from the photolysis of $\mathrm{H}_{2} \mathrm{~S}$. One laser pulse at half the energy of the Lyman- $\alpha$ line of $\mathrm{H}$ serves both to photolyse $\mathrm{H}_{2} \mathrm{~S}$ and to ionize $\mathrm{H}$ atoms via $2+1$ REMPI. (d) The same system as in (c) viewed with polarization as in (b). 
tribution for a parallel transition may be thought of as "polar ice caps." We take two-dimensional images from the three dimensional spatial distribution from viewpoints perpendicular and parallel to the polarization vector of the photolysis laser. Viewed from the side, as in Figure 2a, two "mushroom caps," with a nodal line through the middle are seen. Rotation of the polarization vector of the photolysis laser by $90^{\circ}$ produces an image with radial symmetry and no nodes, as seen in Figure $2 b$.

For a perpendicular transition, the photofragment distribution may be described as an "equatorial belt," as seen in Figures $2 \mathrm{c}$ and $2 \mathrm{~d}$. A $\sin ^{2} \theta$ intensity distribution is produced, which is the complement of that produced for a parallel transition. Figure $2 \mathrm{c}$ shows the image of $\mathrm{H}$ atoms from 243-nm photolysis of $\mathrm{H}_{2} \mathrm{~S}$ with the photolysis laser's polarization vector perpendicular to the detector axis. The intensity pattern is a band with higher intensity at the edges. This edge brightening is simply a consequence of projecting a three-dimensional shell onto a plane. Viewing the same "equatorial belt" with polarization of the photolysis laser parallel to the detector axis produces a "doughnut" as seen in Figure 2d. These four images demonstrate how easily photofragment imaging can determine the type of transition that causes dissociation in the parent molecule.

\section{Extracting information from the images}

One may determine $\beta$ parameters from the images. The threedimensional spatial distribution of photofragments may be reconstructed from an image and this distribution fit to the equation given in the previous section. Alternatively, one may fit only a stripe down the center of the image. This center-stripe technique will be considered in more detail in the section on branching ratios. Photofragment imaging offers subtle advantages over other techniques for determining $\beta$ parameters.

If one measures a $\beta$ parameter of 2 or -1 (the limiting cases shown in Figure 2) the photofragment dynamics may be easily understood. The $\beta$ parameter usually falls somewhere between these two extremes, and hence some interpretation is required. Unfortunately, no technique is able to distinguish between the situation in which a photodissociation occurs through one state with mixed perpendicular and parallel character versus the case where two states (one perpendicular, and one 
parallel) are involved in photodissociation at a given wavelength unless the two states produce fragments with different internal state distributions. Consider two states of the same energy, but different symmetry, both leading to a dissociation.

Let: $X=$ the fraction of fragments arising from a pure parallel transition $(\beta=2)$.

$(1-X)=$ contribution fraction from a pure perpendicular transition $(\beta=-1)$.

Then the distribution of photofragments produced from these two states will be described by:

$$
\mathrm{I}_{\mathrm{two}}(\theta)=\left[1+(3 X-1) P_{2}(\cos \theta)\right] / 4 \pi \text {. }
$$

Therefore, this mixture of fragments arising from two states will be indistinguishable from one state with an intermediate $\beta^{\prime}=(3 X-1)$. This argument assumes the experiment averages over final $m_{J}$ levels. ${ }^{30}$ This analysis considers only the situation in which the photofragments have the same translational energy. In the case where photofragments have different speeds, such as for $\mathrm{HI} \rightarrow \mathrm{H}+\mathrm{I},{ }^{11}$ photofragment imaging can distinguish different $\beta$ parameters from the different channels.

Where two (or more) product states are possible, photofragment imaging may be used to determine the branching ratio. Branching ratios may be determined using other techniques, but a knowledge of the $\beta$ parameter for each channel is required. With photofragment imaging, branching ratios and $\beta$ parameters may be determined at the same time. Consider the photodissociation of $\mathrm{CD}_{3} \mathrm{I}$ to produce $\mathrm{CD}_{3}+\mathrm{I}$. Two dissociation channels are possible, one to produce ground state iodine, $\mathrm{I}\left({ }^{2} P_{3 / 2}\right)$, and a second to produce electronically excited iodine, $I^{*}\left({ }^{2} P_{1 / 2}\right)$. These channels will produce different $\mathrm{CD}_{3}$ translational energies because the internal energy of the $\mathrm{I}$ atom is different and energy must be conserved:

$$
E_{\text {int }}\left(\mathrm{CD}_{3} \mathrm{I}\right)+E_{\mathrm{h} v}=\mathrm{D}_{0}\left(\mathrm{I}-\mathrm{CD}_{3}\right)+E_{\text {int }}(\mathrm{I})+E_{\text {int }}\left(\mathrm{CD}_{3}\right)+E_{\text {trans }} .
$$

Momentum conservation may be used to derive an expression for the speed of the $\mathrm{CD}_{3}$ fragment:

$$
v\left(\mathrm{CD}_{3}\right)=\left\{2 E_{\text {trans }} / m\left(\mathrm{CD}_{3}\right)\left[1+m\left(\mathrm{CD}_{3}\right) / m(\mathrm{I})\right]\right\}^{1 / 2} .
$$

In these equations, $E$ is energy, $m$ is mass, and $v$ is speed. On the 
image, where a single $\mathrm{CD}_{3}$ state has been projected, the two channels will appear as an inner (slow $\mathrm{CD}_{3}$ ) ring, corresponding to the $\mathrm{I}^{*}$ channel, and an outer (fast $\mathrm{CD}_{3}$ ) ring, corresponding to the I channel. Examples are shown in Figure 3. Figure 3a shows an image of $\mathrm{CD}_{3}$ with 2 quanta of $v_{2}$, while Figure $3 \mathrm{~b}$ shows an image from $\mathrm{CD}_{3}$ with 3 quanta of $v_{2}$. The branching ratio $I^{*} /\left(I^{*}+I\right)$ is dramatically different for these two vibrational states. $I^{*} /\left(I^{*}+I\right)=0.89 \pm 0.04$ for Figure $3 a$, while $I^{*} /\left(I^{*}+I\right)=0.54 \pm 0.06$ for Figure $3 b$. Figure $2 a$, which shows $C D_{3}$ probed in the ground vibrational state, was chosen because the branching ratio is nearly 1.0 , so there is no outer ring to complicate the figure.

There are several ways to calculate the branching ratio from photofragment images. The most accurate method is to perform a threedimensional reconstruction of the distribution, and to sum the intensity in the shells of fragments of the same radius. Each shell corresponds to a speed, hence two shells are obtained for $\mathrm{CD}_{3}$ fragments from photolysis of $\mathrm{CD}_{3} \mathrm{I}$. The primary advantage of this method is that it is independent of the $\beta$ parameter for the two channels. An additional attractive feature is that information usually obtained via Doppler spectroscopy is obtained without the use of sub-Dopplerlinewidth lasers. A minor drawback of the three-dimensional reconstruction technique is that a large computational effort is required.

Another option is to examine only the intensity pattern of a stripe down the center of an image. This is equivalent to looking at a plane of the three-dimensional spatial distribution, and collapsing this plane to a line. Note that spinning this plane about the center axis will regenerate the entire three-dimensional distribution, so no information is lost in this process. The intensity distribution of this center stripe may be fit to determine $\beta$ parameters and branching ratios. The intensity distribution along the center stripe is described by:

$$
\mathrm{dI}(x) / \mathrm{d} x=\left(\beta r^{2}-2 r^{2}-3 \beta x^{2}\right) /\left[(8 \pi r)\left(r^{2}-x^{2}\right)^{1 / 2}\right],
$$

where $r$ is the radius of the image, and $x$ ranges from $-r$ to $r$. $\mathrm{I}(x)$ is a continuous function that must be integrated over a small range corresponding to the detector resolution to obtain the intensity at a given pixel on the detector. For two dissociation channels, one fits the sum of two intensity distributions with different values of $r$. The peaks in the center stripe intensity profiles are more pronounced than in Doppler profiles, hence branching ratios may be determined more reliably. An 

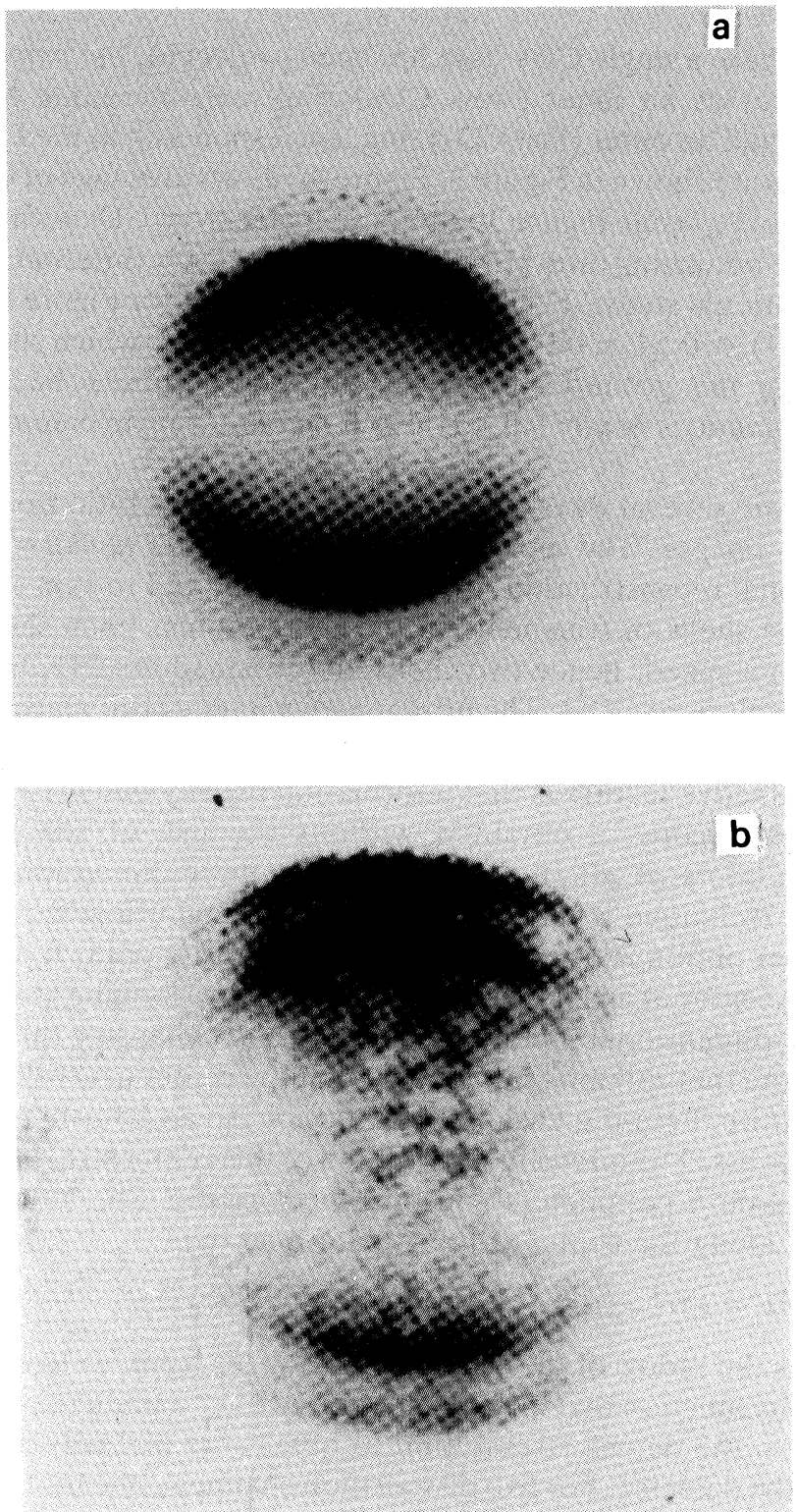

Figure 3 Images of $\mathrm{CD}_{3}$ from 266-nm photolysis of $\mathrm{CD}_{3} \mathrm{I}$ to show the different branching ratios as a function of $\mathrm{CD}_{3}$ vibrational level. (a) Image recorded with the laser's wavelength set at $339.36 \mathrm{~nm}\left(\mathrm{CD}_{3} 2_{2}^{0}\right.$ band $) . I^{*} /\left(I^{*}+\mathrm{I}\right)=0.89 \pm 0.04$. (b) Image recorded with the laser's wavelength set at $324.37 \mathrm{~nm}\left(\mathrm{CD}_{3} 2_{3}^{3}\right.$ band $) . \mathrm{I}^{*} /\left(\mathrm{I}^{*}+\mathrm{I}\right)=0.53 \pm$ 0.06 . 
additional advantage to the center-stripe technique is that data may be recorded with a (less expensive, and often more readily available) one-dimensional detector. The two-dimensional image is seen on the phosphor screen, but only the intensity along a center stripe needs to be collected with a CCD detector.

Other techniques may be used to determine branching ratios (and $\beta$ parameters). Summing the pixels of a two-dimensional image parallel to the photolysis laser's polarization axis produces a one-dimensional projection that is equivalent to a Doppler profile. Branching ratios and $\beta$. parameters are then obtained in the usual manner. ${ }^{10-12}$ The onedimensional projection obtained from a photofragment image is equivalent to the one-dimensional projection of the velocity distribution obtained in time of flight experiments. ${ }^{13,18,19}$

Other research groups have derived information from photofragment distributions by examining a "core" of the three-dimensional distribution. Koplitz et al. ${ }^{20}$ employ a clever variation of Doppler spectroscopy where the probe laser is delayed in time with respect to the photolysis laser so that it samples only fragments that have no velocity component perpendicular to the laser axis. Krautwald et al. ${ }^{19}$ tag photofragments with REMPI and extract the ions through a small hole into a time-of-flight apparatus, thus obtaining a one-dimensional sample of the three-dimensional velocity distribution. Loo et al. ${ }^{13}$ describe a variation of the TOF coring whereby the fragments' velocity perpendicular to the detector axis carry them out of the detector region. The TOF coring technique has no equivalent in photofragment imaging at present. The intensity pattern of peaks in the TOF coring technique will show sharper peaks than the center-stripe technique described here, thus branching ratios should be even simpler to determine. The TOF coring technique, however, requires prior knowledge of the $\beta$ parameter for each branching channel that is being investigated. For example, if the polarization axis of the dissociation laser was parallel to the detector axis and a parallel channel and a perpendicular channel were being investigated, the TOF coring would not detect fragments from the perpendicular channel.

\section{Doppler profile component selection}

For $\mathrm{CD}_{3}$ fragments produced by photodissociation of $\mathrm{CD}_{3} \mathrm{I}$ at $266 \mathrm{~nm}$, the Doppler width $\left(\sim 2 \mathrm{~cm}^{-1}\right)$ created by the fragment recoil velocity is 
not completely covered by the bandwidth of the ionization laser. This potential problem is easily overcome by scanning the frequency of the ionization laser as described in the experimental section. If we sum the intensity pattern of the images obtained in this manner by rows or by columns (i.e. collapse the two-dimensional image to one dimension) we obtain the equivalent of a Doppler profile without the use of sub-Doppler-linewidth lasers. This summation technique is fully equivalent to the one-dimensional TOF technique, where this advantage has been recognized by Loo et al. ${ }^{13}$ and by Black. ${ }^{18}$ The photofragment imaging technique used in this way can take advantage of the analysis mathematics developed by other researchers. ${ }^{10-12}$

One must be careful when using TOF or imaging techniques that the bandwidth of the ionization laser covers the entire Doppler profile of the species being ionized, or else the $\beta$ parameter may be incorrectly determined. This problem is illustrated in Figure 4 for the dissociation of $\mathrm{H}_{2} \mathrm{~S}$ and imaging of the $\mathrm{H}$ atom fragment. Figures $4 \mathrm{a}-\mathrm{c}$ were collected with the laser at a fixed wavelength so that only a small component of the Doppler profile was imaged. Each subset of the velocity profile transforms to a stripe on the two-dimensional detector. One could not, without prior knowledge of the system, hope to derive information about the dynamics of the photodissociation from such an image. This problem is overcome by scanning the probe laser in frequency while the image is being collected. Figure $4 \mathrm{~d}$ shows the image produced when the photolysis laser is scanned over the entire Doppler profile of the $\mathrm{H}$-atom Lyman- $\alpha$ transition.

Again we point out the advantages of the center-stripe technique in determining $\beta$ parameters and branching ratios. An image may be collected while keeping the probe laser's frequency at the center of the Doppler profile (not scanning the laser). An analysis of the intensity pattern of a stripe down the middle of the image produced will provide $\beta$ parameters and branching ratios without concern for the wings of the Doppler profile. Note that the wings of the Doppler profile transform to the sides of the image when the laser propagation axis is horizontal. A one-dimensional array detector may also be employed in this configuration.

\section{Polarization effects in photofragment images}

Houston has reviewed ${ }^{31}$ how vector correlations effect the three- 

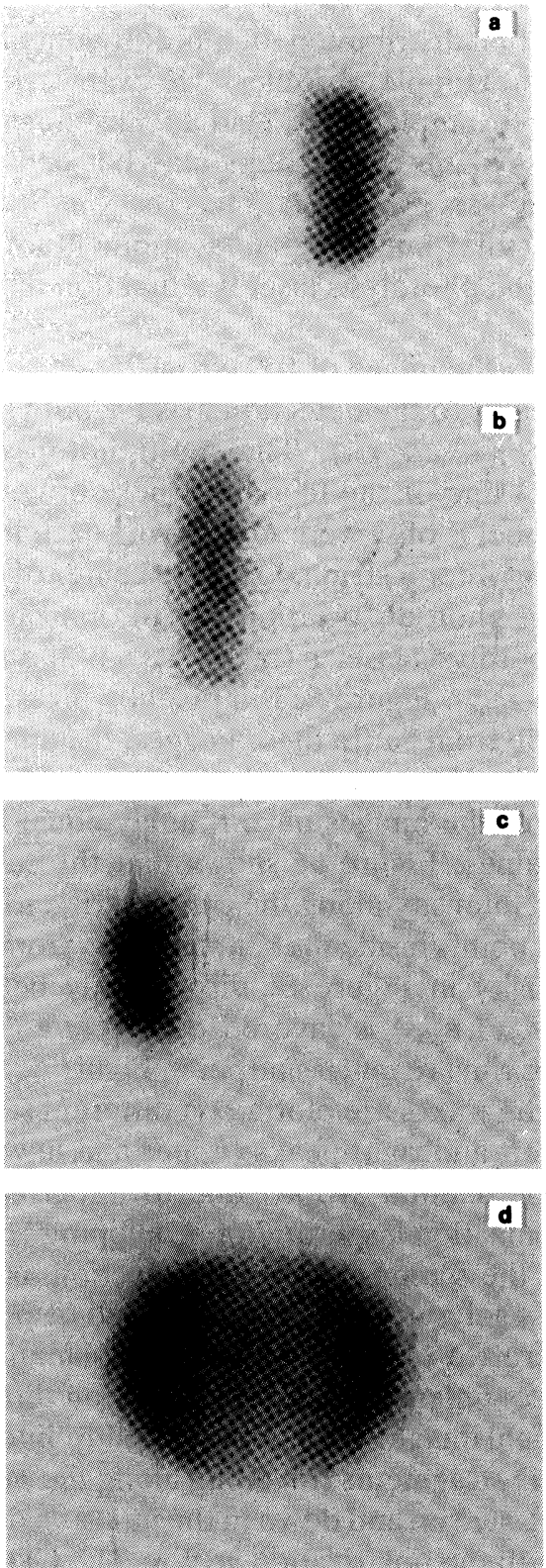

Figure 4 Images of $\mathrm{H}$ atoms from the $243 \mathrm{~nm}$ photolysis of $\mathrm{H}_{2} \mathrm{~S}$. One laser is used for both photolysis and ionization of $H$ using $2+1$ REMPI via $H$ Lyman- $\alpha$. (a) Image recorded with the laser's frequency set at $\sim 1 / 2 * 82266 \mathrm{~cm}^{-1}$. (b) Image recorded with the laser's frequency set at $\sim 1 / 2 * 82261 \mathrm{~cm}^{-1}$. (c) Image recorded with the laser's frequency set at $\sim 1 / 2 * 82259 \mathrm{~cm}^{-1}$. (d) Image recorded while scanning the laser's frequency from $\left(1 / 2 * 82250 \mathrm{~cm}^{-1}\right)$ to $\left(1 / 2 * 82270 \mathrm{~cm}^{-1}\right)$. 
dimensional spatial distribution of photofragments and how these effects appear in Doppler profiles. He warns that neglect of v-J correlations, for example, may lead to misinterpretation of the $\beta$ parameter. These same correlations may be seen in photofragment images. An example is presented in this section for $\mathrm{CD}_{3}$. We find that alignment of $\mathbf{N}$ (total nuclear angular momentum) with the polarization vector of the photolysis laser is a function of the velocity of the fragment.

Figure 5a shows the image of $\mathrm{CD}_{3}$ from 266-nm photolysis of $\mathrm{CD}_{3} \mathrm{I}$ with $\varepsilon$ perpendicular to the detection axis for both the photolysis and ionization lasers $\left(\varepsilon_{\text {ion }} / / \varepsilon_{\text {phot }}\right)$. The multiphoton ionization occurs through the $\mathrm{R}(2)$ line of the $0_{0}^{0}$ band where only the adiabatic (I*) dissociation channel is observed. Overlaying each image is a graph of the intensity pattern for a stripe down the center of the image. Figure 5 b shows the same photodissociation with the ionization laser $\varepsilon$ perpendicular to the photolysis laser $\varepsilon\left(\varepsilon_{\text {ion }} \perp \varepsilon_{\text {phot }}\right)$, and the detection axis perpendicular to $\varepsilon_{\text {phot }}$. Figure $5 \mathrm{c}$ is a pixel by pixel subtraction of the image in Figure $5 \mathrm{~b}$ from $5 \mathrm{a}$. In these images, the upper and lower rims of the circle are $25 \%$ brighter with $\varepsilon_{\text {ion }} / / \varepsilon_{\text {phot }}$ than with $\varepsilon_{\text {ion }} \perp \varepsilon_{\text {phot }}$. The middle portions of the images have roughly equal intensity for both polarization schemes. Figure $5 \mathrm{c}$ shows that the subtraction process gives nearly zero intensity along the center stripe for all locations but the outer rim. At points inside the rim, but away from the middle of the image, the intensity in Figure $5 \mathrm{c}$ dips slightly below the baseline, indicating that $\varepsilon_{\text {ion }} \perp \varepsilon_{\text {phot }}$ is favored over $\varepsilon_{\text {ion }} / / \varepsilon_{\text {phot }}$. Note that the peaks at the outer rim are much sharper in Figure $5 \mathrm{c}$ than in Figures 5a and $5 \mathrm{~b}$ and that the $y$ axes are plotted on different scales. The relative intensity of the left and right peaks (upper and lower portions of the image) is an experimental artifact due to imperfect overlap of the photolysis and ionization lasers and/or a "clipping" of a portion of the image by one of the screens along the detection axis.

A quantitative analysis of the anisotropy will be presented in a later publication $;{ }^{32}$ here we offer a qualitative explanation. Consider the molecules in three dimensions and the effect on different portions of the two-dimensional image. We assume a pseudo-linear dissociation of the $\mathrm{CD}_{3} \mathrm{I}$. The $\mathrm{CD}_{3}$ fragments travelling along the polarization vector of the photolysis laser (up and down in the images), are born with their $\mathrm{C}_{3}$ axis aligned with the polarization vector. The $\mathrm{CD}_{3}$ can tumble, but this tumbling will not completely wash out the initial alignment. 

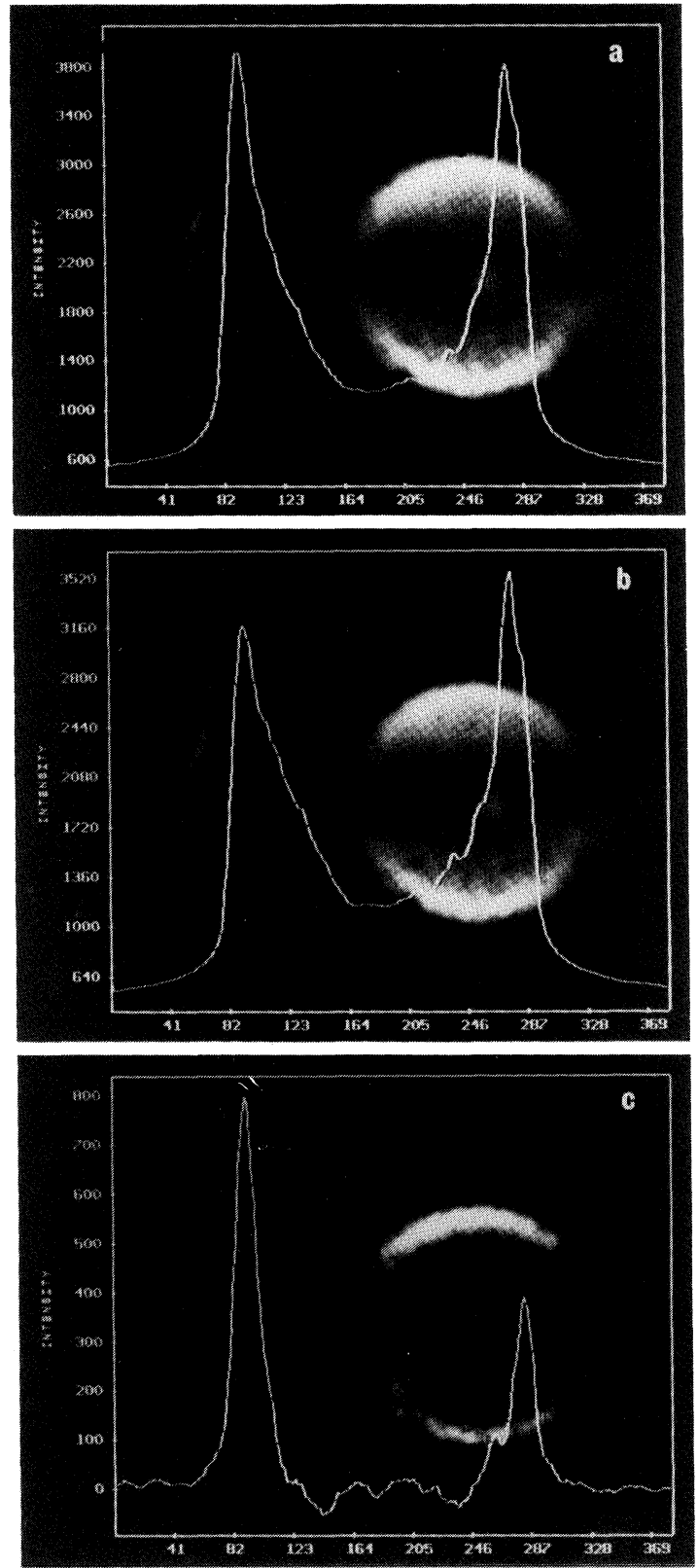

Figure 5 Images of $\mathrm{CD}_{3}$ via REMPI through the $0_{0}^{0} \mathrm{R}(2)$ band from the 266-nm photolysis of $\mathrm{CD}_{3} \mathrm{I}$. All images were collected with the photolysis laser's polarization vector, $\varepsilon_{\mathrm{phot}}$, perpendicular to the detection axis. Overprinting each image is a plot of the intensity vs. pixel number for a stripe through the center of the image. (a) Image collected with the ionization laser's polarization vector parallel to the photolysis laser's polarization vector $\left(\varepsilon_{\text {ion }} / / \varepsilon_{\text {phot }}\right)$. (b) Image collected with $\varepsilon_{\text {ion }} \perp \varepsilon_{\text {phot }}$. (c) Pixel by pixel subtraction of image (b) from image (a). 
Hence, $\varepsilon_{\text {ion }} / / \varepsilon_{\text {phot }}$ will be preferentially absorbed. Classically, $\mathrm{CD}_{3}$ radicals may undergo three rotational motions; a helicopter motion about the $\mathrm{C}_{3}$ axis, and two tumbling motions. The two tumbling motions will decrease the amount of absorption, but $\varepsilon_{\text {ion }} / / \varepsilon_{\text {phot }}$ will still be favored over $\varepsilon_{\text {ion }} \perp \varepsilon_{\text {phot }}$. When molecules travel toward or away from the detector (seen in the center of the images) $\mathbf{N}$ will be oriented along the detection axis, hence $\varepsilon_{\text {ion }} \perp \varepsilon_{\text {phot }}$ will be favored. On the center stripe intensity, we see a slight preference for $\varepsilon_{\text {ion }} \perp \varepsilon_{\text {phot }}$ near the middle of the image.

If there were no alignment, then there would not be any preferential absorption of a linearly polarized ionization laser, and the subtracted image would show zero intensity throughout. For $\mathrm{CD}_{3}$ fragments from $\mathrm{CD}_{3} \mathrm{I}$ photolysis, we observe increasing alignment with increasing $\mathbf{N}$. The images shown in Figure 5 are taken with REMPI through the R(2) line of the origin band. In another paper, ${ }^{22}$ we show images taken with REMPI through the $R(5)$ line of the origin band. With higher $\mathbf{N}$ we see a larger preference for $\varepsilon_{\text {ion }} / / \varepsilon_{\text {phot }}$ on the upper and lower rims of the image. For $\mathbf{N}=2$, the upper edge of the subtracted image has $1 / 4$ the intensity of the $\varepsilon_{\text {ion }} / / \varepsilon_{\text {phot }}$ image, while for $\mathbf{N}=5$ the upper edge of the subtracted image has $1 / 2$ the intensity of the $\varepsilon_{\text {ion }} / / \varepsilon_{\text {phot }}$ image.

\section{CONCLUSION}

We have demonstrated the power of the photofragment imaging technique by applying the technique to the study of two unimolecular reactions. This technique allows one to determine uniquely the spatial distribution of the fragments from a photodissociation event. The spatial distribution of the fragments contains information regarding: the absorption process of the parent molecule (parallel or perpendicular transitions), the lifetime of the parent molecule (through the $\beta$ parameter), and the partitioning of energy during the dissociation process (through the velocity of the state-selected fragments). Because of the selectivity of the REMPI process, we obtain spatial information as a function of internal state of the fragment. Finally, the technique allows us to obtain information about the alignment of the fragment molecular axis with respect to the laser polarization axis.

The technique of ion imaging is not, however, limited to the study of photofragmentation. Ion imaging should prove generally applicable whenever multiphoton ionization can be used to detect the products of 
a bimolecular chemical process where the initial reagent velocity is specified. Examples of such processes include crossed-molecularbeam reactions, beam-gas reactions, and photoinitiated reactions within clusters. We hope to demonstrate the utility of ion imaging to bimolecular reactions in the near future.

\section{Acknowledgements}

It is our pleasure to thank Mark Jaska and Mitch Williams for their expert technical assistance.

\section{References}

1. J. Solomon, J. Chem. Phys. 47, 889 (1967).

2. C. Jonah, P. Chandra and R. Bersohn, J. Chem. Phys. 55, 1903 (1971).

3. J. Solomon, C. Jonah, P. Chandra and R. Bersohn, J. Chem. Phys. 55, 1908 (1971).

4. G. E. Busch, R. T. Mahoney, R. I. Morse and K. R. Wilson, J. Chem. Phys. 51, 449 (1969).

5. R. W. Diesen, J. C. Wahr and S. E. Adler, J. Chem. Phys. 50, 3635 (1969).

6. M. Dzvonik, S. Yang and R. Bersohn, J. Chem. Phys. 61, 4408 (1974).

7. W. M. Jackson, J. Chem. Phys. 59, 960 (1973).

8. W. M. Jackson and R. J. Cody, J. Chem. Phys. 61, 4183 (1974).

9. A. P. Baronavski and J. R. McDonald, Chem. Phys. Lett. 45, 172 (1977).

10. J. L. Kinsey, J. Chem. Phys. 66, 2560 (1977).

11. R. Schmiedl, H. Dugan, W. Meier and K. H. Welge, Z. Phys. A 304, 137 (1982).

12. I. Nadler, D. Mahgerefteh, H. Reisler and C. Wittig, J. Chem. Phys. 82, 3385 (1985).

13. R. O. Loo, G. E. Hall, H.-P. Haerri and P. L. Houston, submitted.

14. D. Eres, M. Grunick and J. D. McDonald, J. Chem. Phys. 81, 5552 (1984).

15. D. W. Chandler and P. L. Houston, J. Chem. Phys. 87, 1445 (1987).

16. W. C. Wiley and I. H. McLaren, Rev. Sci. Instrum. 26, 1150 (1955).

17. K. R. Castleman, Digital Image Processing (Prentice-Hall, Englewood Cliffs, 1979), pp. 184-185.

18. J. F. Black, Ph.D. Thesis, University of Nottingham (1987); J. F. Black and I. Powis, J. Chem. Phys., submitted.

19. H. J. Krautwald, L. Schnieder, K. H. Welge and M. N. R. Ashfold, Faraday Discuss. Chem. Soc. 82, 99 (1986).

20. B. Koplitz, Z. Xu, D. Baugh, S. Buelow, D. Hausler, J. Rice, H. Reisler, C. X. W. Qian, M. Noble and C. Wittig, Faraday Discuss. Chem. Soc. 82, 125 (1986).

21. J. W. Hudgens, T. G. DiGiuseppe and M. C. Lin, J. Chem. Phys. 79, 571 (1983).

22. D. W. Chandler, J. W. Thoman, Jr., M. H. M. Janssen and D. H. Parker, manuscript in preparation.

23. G. N. A. van Veen, T. Baller, A. E. de Vries, and N. J. A. van Veen, Chem. Phys. 87, 405 (1984).

24. G. N. A. van Veen, K. A. Mohamed, T. Baller and A. E. de Vries, Chem. Phys. 74, 261 (1983).

25. R. N. Zare and D. R. Herschbach, Proc. IEEE 51, 173 (1963).

26. R. Bersohn and S. Lin, Adv. Chem. Phys. 16, 67 (1969).

27. C. Jonah, J. Chem. Phys. 51, 1915 (1971). 
28. S. Yang and R. Bersohn, J. Chem. Phys. 61, 4400 (1974).

29. R. N. Dixon, Mol. Phys. 54, 333 (1985).

30. M. Shapiro and R. Bersohn, Ann. Rev. Phys. Chem. 33, 409 (1982).

31. P. L. Houston, J. Phys. Chem. 91, 5388 (1987).

32. M. H. M. Janssen, S. Stolte, J. W. Thoman, Jr., D. W. Chandler and D. H. Parker, manuscript in preparation. 